\title{
Clinicopathological features of recurrent papillary thyroid cancer
}

\author{
Jian Zhu' ${ }^{1 \dagger}$, Xinli Wang ${ }^{2 \dagger}$, Xiaoxuan Zhang ${ }^{3 \dagger}$, Peifeng $\mathrm{Li}^{4^{*}}$ and Haifeng $\mathrm{Hou}^{5}$
}

\begin{abstract}
Background: To investigate the clinicopathological features of recurrent papillary thyroid carcinoma (PTC).

Methods: A retrospective analysis on clinical and pathological data of 34 patients with recurrent PTC was carried out. A total of 281 patients with non-recurrent PTC during the same time period were chosen as the control group.

Results: Patients were divided into three groups according to the pathological subtype. The number of patients belonging to Groups 1, 2, and 3 were 28, 154, and 133, respectively. 78 patients underwent partial or whole thyroidectomy, 151 cases underwent thyroidectomy combining neck regional lymph node dissection, and 86 patients underwent thyroidectomy combining modified or radical neck dissection. Univariate analysis showed that PTC recurrence was associated with tumor size, extrathyroid invasion, initial surgery approach, lymph node metastasis, and pathological subtype $(P<0.05)$. Patient age, gender, complication with Hashimoto's thyroiditis, and multifocality were unrelated to PTC recurrence $(P>0.05)$. Multivariate analysis showed that initial surgery approach and pathological subtype perform important functions in PTC recurrence $(P<0.001)$. Initial surgery approach presented a negative correlation with PTC recurrence $(\beta=-0.320, O R=0.726)$. The pathological subtype was also related to PTC recurrence $(\beta=0.923, \mathrm{OR}=2.517)$.

Conclusion: PTC patients without neck dissection showed greater likelihood of postoperative recurrence. Patients with the tall cell, columnar cell, diffuse sclerosing, and oncocytic variants showed a higher propensity for PTC recurrence after operation compared with those who did not. Tumor volume, extrathyroid invasion, and multiple lymph node metastases at the time of initial operation were also significantly related to postoperative recurrence. Follow-up supervision must be enhanced after initial treatment to mitigate PTC recurrence in susceptible patients. Effective and standard treatments must be adopted immediately after the discovery of recurrence.
\end{abstract}

Keywords: Clinicopathological features, Initial surgery approach, Papillary thyroid cancer, Pathological subtype, Recurrence

\section{Background}

Papillary thyroid carcinoma (PTC) is the most common thyroid cancer; this cancer presents relatively low malignancy, good prognosis, and a 10-year survival rate of over $90 \%$. However, the clinical behaviors of this cancer are complex and varied. PTC is easy to spread via lymphatic ducts, which results in recurrence, metastases, and even death [1]. Recurrent PTC mainly refers to localized and distant recurrence, including recurrence of the primary tumor, lymph node metastases, invasion of

\footnotetext{
* Correspondence: lipeifeng00@hotmail.com

†Equal contributors

${ }^{4}$ Department of Pathology, General Hospital of Jinan Military Command, 25

Shifan Road, Tianqiao District, Jinan 250031, China

Full list of author information is available at the end of the article
}

the esophagus and trachea, invasion of muscles, soft tissues and nerves, and distant metastases. Many factors can affect the recurrence of thyroid cancer, and final conclusion has not yet been reached. Many researchers believe that pathological type, staging, degree of extrathyroid invasion, lymph node metastatic rate, age, and initial surgery approach may be related to thyroid cancer recurrence. This research retrospectively analyzes the clinical data of 34 patients with recurrent PTC and presents a statistical comparison with non-recurrent patients to analyze factors related to PTC recurrence. The aim of the present work is to provide clinical evidence for standardized and appropriate treatment of recurrent PTC.

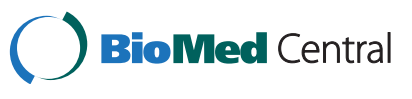

(c) 2015 Zhu et al. This is an Open Access article distributed under the terms of the Creative Commons Attribution License (http://creativecommons.org/licenses/by/4.0), which permits unrestricted use, distribution, and reproduction in any medium, provided the original work is properly credited. The Creative Commons Public Domain Dedication waiver (http:// creativecommons.org/publicdomain/zero/1.0/) applies to the data made available in this article, unless otherwise stated. 


\section{Methods}

\section{Clinical data}

A total of 315 patients with PTC who were admitted to Jinan Military General Hospital from January 2009 to July 2013, including 66 male patients and 249 female patients, were recruited. The male to female ratio was 1:3.8. Ages ranged from 20 years to 78 years, and the median age was 48 years. The diameter of most tumors was less than $2 \mathrm{~cm}$, the proportion of which was $47.1 \%$ in the recurrent group and $85.8 \%$ in the non-recurrent group. Moreover, $10.5 \%$ (33 cases) of the patients presented extrathyroid invasion, and $42.2 \%$ (133 cases) of the patients were found with lymph node metastases at the time of initial surgery. The tumors of $44.4 \%$ (140 cases) of the patients showed multifocality, and $25.1 \%$ (79 cases) of the patients had Hashimoto's thyroiditis (HT) (Table 1). This study received the approval of the ethics committee of the General Hospital of Jinan Military Command (No. 2013ZD01).

\section{Pathological subtype of PTC}

Recruited PTC patients were divided into three groups. Patients with the tall cell, columnar cell, diffuse sclerosing, and oncocytic variants with higher invasiveness, higher probability of recurrence, and metastases were classified into Group 1. Patients with the follicular, clear cell, and conventional papillary carcinoma variants with similar prognosis were classified into Group 2. Patients with papillary microcarcinoma were classified into Group 3.

\section{Initial surgery approach}

The methods of primary lesion dissection used in this research included thyroid lobectomy with or without isthmectomy and total/near-total thyroidectomy. The methods of lymph node dissection included central compartment node dissection, selective neck dissection, modified neck dissection, and radical neck dissection. During statistical analysis, thyroid lobectomy with or without isthmectomy and total/near-total thyroidectomy were classified as the first type of surgical approach. Surgical approaches belonging to the first type combined with central compartment node dissection, selective neck dissection, and wide dissection of metastatic lesions were classified as the second type of surgical approach. Finally, approaches combining modified neck dissection and radical dissection were classified as the third type of surgical approach.

\section{Statistical analysis}

SPSS 17.0 software was used for statistical analysis. In addition, $\chi^{2}$-test and multivariate analysis using Cox proportional hazards modeling were carried out. Factors that may be related to PTC recurrence, including gender,
Table 1 Clinicopathological features of patients with recurrent and non-recurrent PTC

\begin{tabular}{|c|c|c|c|c|}
\hline $\begin{array}{l}\text { Clinicopathological } \\
\text { feature }\end{array}$ & $\begin{array}{l}\text { Recurrent } \\
\text { group } \\
(N=34)\end{array}$ & $\begin{array}{l}\text { Non-recurrent } \\
\text { group } \\
(N=281)\end{array}$ & $x^{2}$ & $P$ \\
\hline Age/year & & & 0.32 & 0.574 \\
\hline$<45$ & 14 & 130 & & \\
\hline$\geq 45$ & 20 & 151 & & \\
\hline Gender & & & 3.11 & 0.078 \\
\hline Male & 11 & 55 & & \\
\hline Female & 23 & 226 & & \\
\hline Tumor size & & & 34.76 & $<0.001$ \\
\hline$<1$ & 3 & 130 & & \\
\hline$\geq 1-<2$ & 13 & 111 & & \\
\hline$\geq 2$ & 18 & 40 & & \\
\hline Initial surgery approach & & & 11.39 & 0.003 \\
\hline First type & 16 & 62 & & \\
\hline Second type & 14 & 137 & & \\
\hline Third type & 4 & 82 & & \\
\hline Extra thyroidal invasion & & & 22.15 & $<0.001$ \\
\hline Yes & 12 & 21 & & \\
\hline No & 22 & 260 & & \\
\hline $\begin{array}{l}\text { Coexistent Hashimoto's } \\
\text { thyroiditis }\end{array}$ & & & 0.38 & 0.537 \\
\hline Yes & 10 & 69 & & \\
\hline No & 24 & 212 & & \\
\hline Lymphatic metastasis & & & 10.10 & 0.001 \\
\hline Yes & 23 & 110 & & \\
\hline No & 11 & 171 & & \\
\hline Multifocality & & & 0.00 & 0.968 \\
\hline Yes & 15 & 125 & & \\
\hline No & 19 & 156 & & \\
\hline Pathological subtype & & & 34.45 & $<0.001$ \\
\hline Group 1 & 11 & 17 & & \\
\hline Group 2 & 20 & 134 & & \\
\hline Group 3 & 3 & 130 & & \\
\hline
\end{tabular}

age, tumor size, initial surgery approach, lymph node metastasis, number of lesions, complication with HT, extrathyroid invasion, and pathological subtype, were evaluated. $P<0.05$ was considered statistically significant difference.

\section{Results}

\section{Clinicopathological information}

The clinicopathological information of PTC patients is given in Table 1, and the comparisons between patients with recurrence and non-recurrence were carried out. 34 recurrent patients (11 males and 23 females) were 
investigated in this study. The male to female ratio was 1:2.1. Ages ranged from 24 years to 72 years old, and the median age was 46 years old. Postoperative recurrence intervals from initial surgery ranged from five months to 18 years, with a median of 46 months. Twelve patients (35.3\%) showed postoperative recurrence within two years, 14 patients $(41.2 \%)$ showed postoperative recurrence within two to five years, and eight patients $(23.5 \%)$ showed postoperative recurrence over five years later. Recurrence occurred once in 29 patients (85.3\%), twice in 4 patients $(11.8 \%)$, and thrice in 1 patient (2.9\%). Two hundred eighty-one non-recurrent PTC patients were classified into the control group (55 males and 226 females); in this group, the male to female ratio was 1:4.1, the age of onset ranged from 20 years to 78 years old, and the median age was 48 years. Patients were divided into three groups according to the pathological subtype. The number of patients belonging to Groups 1, 2, and 3 among the 34 recurrent patients were 11,20 , and 3, respectively. The number of non-recurrent patients belonging to Groups 1, 2, and 3 were 17, 134, and 130 (Table 2), respectively. Among the 34 recurrent patients, 16 patients were treated using surgeries of the first type, 14 patients were treated using surgeries of the second type, and 4 patients were treated using surgeries of the third type. The numbers of non-recurrent patients treated by the first, second, and third types of surgery were 62,137 , and 82 , respectively.

\section{Univariate analysis of factors related to PTC recurrence}

Tumor size, extrathyroid invasion, initial surgery approach, lymph node metastases, and pathological subtype showed statistical differences between the recurrent and non-recurrent groups $(P<0.05$, Table 1$)$. Larger tumors showed significantly increased risk of recurrence. The recurrence rate of micro-PTC was $2.3 \%(3 / 133)$ and reached $31.0 \%(18 / 58)$ when the tumor diameter grew to $\geq 2 \mathrm{~cm}$. The higher recurrence rates, reaching $35.3 \%(12 / 34)$, were observed when extrathyroid invasion was present at the time of initial surgery. Initial surgery approach also showed a significant effect on PTC

Table 2 Pathological subtypes of PTC

\begin{tabular}{lll}
\hline Pathological subtype & Recurrent group & Non-recurrent group \\
\hline ConventionalPTC & 20 & 121 \\
Oncocytic variant & 7 & 11 \\
Clear cell variant & 0 & 4 \\
Follicular variant & 0 & 9 \\
Tall cell variant & 1 & 0 \\
Columnar cell variant & 3 & 4 \\
Diffuse sclerosing variant & 0 & 2 \\
Papillary microcarcinoma & 3 & 130 \\
\hline
\end{tabular}

recurrence; recurrence rates could reach $20.5 \%$ (16/78) in patients without lymph node dissection but only $4.7 \%(4 / 86)$ in patients receiving radical dissection and modified dissection. The recurrence rate was $17.3 \%$ (23/ 133) in patients with cervical lymph node metastasis at the time of initial surgery and only $6.0 \%(11 / 182)$ in patients without metastasis. In this research, patients with the tall cell, columnar cell, and oncocytic variants showed higher recurrence rates than patients with other cell variants (Table 2). Factors such as age, gender, complication with HT, and number of lesions demonstrated no correlation with tumor recurrence $(P>0.05$, Table 1$)$.

\section{Multivariate analysis of factors related to PTC recurrence}

Multivariate analysis revealed that initial surgery approach and pathological subtype were the main factors related to PTC recurrence $(P<0.001$, Table 3$)$. Initial surgery approach was negatively correlated with PTC recurrence $(\beta=-0.320$, OR $=0.726)$. Thus, patients receiving conservative treatment are more likely to develop PTC recurrence than patients treated with more radical modalities. Recurrence rates decreased with increasing surgical scope. Pathological subtype also showed a close correlation with PTC recurrence $(\beta=0.923$, OR $=2.517)$.

\section{Discussion}

PTC is the most common but least aggressive histological subtype of thyroid cancer. Most patients with PTC have excellent prognosis. However, recent studies have demonstrated increasing incidence of recurrent PTC $[2,3]$. Many factors can affect thyroid cancer recurrence, but final conclusions have not been reached. The results of some studies show that the pathological type, staging, degree of extrathyroid invasion, lymph node metastatic rate, age, and initial surgery approach are related to thyroid cancer recurrence $[4,5]$. Our research showed that tumor size, extrathyroid invasion, initial surgery approach, lymph node metastasis, and pathological subtype demonstrate statistically significant differences between the recurrent and non-recurrent groups. By contrast, factors such as age, gender, complication with HT, and number of lesions are not correlated with tumor recurrence. Multivariate analysis results further revealed that initial surgery approach and pathological subtype are main factors related to PTC recurrence.

The dissection methods of primary lesions of PTC include (1) hemithyroidectomy with or without isthmectomy, (2) total/near-total thyroidectomy, and (3) extension of surgical scope. In case of severe extrathyroid invasion, such as invasion of the esophagus, trachea, and nerves, extending the scope of surgery is required. The methods of neck dissection are as follows: (1) central compartment node dissection (unilateral or bilateral), (2) selective neck 
Table 3 Multivariate analysis of factors related to recurrent PTC

\begin{tabular}{lllllll}
\hline Clinicopathological characteristic & $\beta$ & SE & Wald $x^{2}$ & $P$ & OR & $95 \% \mathrm{Cl}$ \\
\hline Initial operative approach & -0.320 & 0.093 & 22.593 & $<0.001$ & 0.726 & $0.605 \sim 0.871$ \\
Pathological subtype & 0.923 & 0.196 & 18.337 & $<0.001$ & 2.517 & $1.714 \sim 3.696$ \\
\hline
\end{tabular}

dissection, (3) functional compartmental en-bloc neck dissection, (4) modified neck dissection, and (5) radical neck dissection [6]. Mazzaferri et al. [7] found that the recurrence rate after partial thyroidectomy is nearly twice that of total and near-total thyroidectomy. By contrast, Cunningham et al. [8] revealed that the recurrence rates had no significant difference between hemithyroidectomy and total/near-total thyroidectomy groups. Monacelli et al. [9] suggested that total thyroidectomy combined with central node dissection must be performed even in the absence of risk factors and without clinically evident nodes. However, some researchers do not advocate prophylactic central neck lymphadenectomy [10]. Univariate analysis in this research showed that initial surgery approach exerts a great impact on the prognosis. For example, while $47.1 \%(16 / 34)$ of the recurrent PTC patients received the first type of surgery as initial surgery, only $22.1 \%(62 / 281)$ of the non-recurrent PTC patients received the first type of surgery. About $52.9 \%$ (18/34) of the recurrent PTC patients received the second and third types of surgery. In comparison, $77.9 \%(219 / 281)$ of the non-recurrent PTC patients received the second and third types of surgery as the initial surgery. Multivariate analysis revealed that initial surgery approach is the main factor related to PTC recurrence $(P<0.001)$; specifically, initial surgery approach demonstrated a negative correlation with PTC recurrence $(\beta=-0.320$, $\mathrm{OR}=0.726$ ).

The recurrence rate decreased with increasing surgical scope. Non-standardized surgical approaches with inappropriately small surgical scopes could lead to tumor residue. Moreover, lesions of lymph node metastasis may be missed, thereby increasing the risk of recurrence. Possible reasons behind the inconsistency of results are as follows: (1) Differences among recruited patients. Patients receiving neck dissection showed significant metastases, whereas no cervical lymph node metastasis was discovered before the operation in patients who did not receive neck dissection. (2) Insufficient number of recruited patients. (3) Difference in surgical techniques among surgeons. Considering these factors, blindly extending or narrowing the surgical scope is irrational.

The World Health Organization (WHO) histological classification of tumors has redefined the subtypes of non-conventional PTC [11]: follicular variant, oncocytic variant, diffuse sclerosing variant, tall cell variant, columnar cell variant, solid variant, PTC with nodular fasciitis-like stroma, clear cell variant, and diffuse follicular variant. PTC patients of different histological subtypes may exhibit diverse clinical and biological behaviors. Subtypes including the tall cell, columnar cell, diffuse sclerosing, and oncocytic variants have higher invasiveness and may promote higher risks of recurrence and metastases [12-14]. The prognosis of patients with the follicular and clear cell variants is similar to that of patients with conventional papillary carcinoma [15]. Thyroid microcarcinoma is a type of papillary carcinoma that is less than $1 \mathrm{~cm}$ in diameter with relatively low invasiveness and good prognosis [16]. This research classified histological variants according to the WHO histological classification of tumors. Patients with the tall cell, columnar cell, diffuse sclerosing, and oncocytic variants were classified into Group 1; patients with the follicular, clear cell, and conventional PTC variants were classified into Group 2; and patients with papillary microcarcinomas were classified into Group 3. The research conducted by Boone et al. [17] showed that the recurrence rate of patients with differentiated thyroid carcinoma is lower than that of patients with other types of thyroid carcinoma. Among the differentiated thyroid carcinomas, the recurrence rate of PTC is lower than that of the follicular variant. However, $30 \%$ of the PTC patients continue to suffer from recurrence, metastasis, and even death [18]. Univariate analysis demonstrated that pathological subtype is obviously correlated with PTC recurrence $(P<0.01)$. Multivariate analysis also indicated that pathological subtype is closely related to PTC recurrence $(\beta=0.923, \mathrm{OR}=2.517)$. The recurrence rate of PTC increased as the invasiveness of the tumor increased. Thus, close follow-up must be carried out in patients with the tall cell, columnar cell, diffuse sclerosing, and oncocytic variants. Effective treatment measures must be taken once recurrence is discovered.

Various results are reported in the literature regarding the effect of lymph node metastases on PTC recurrence. Some studies indicate that lymph node metastases do not affect PTC recurrence [19]. However, some researchers have found that the number of lymph node metastases is associated with postoperative recurrence or re-metastasis. Thus, lymph node metastasis has become an important factor affecting the prognosis and recurrence of thyroid carcinoma [20]. The results of this research revealed statistically significant differences in lymph node metastases between the recurrent and non- 
recurrent groups. Patients with lymph node metastases at the time of initial surgery are more likely to suffer recurrence than those without metastases. The correlation of cervical lymph node metastases with recurrence needs to be confirmed through large-sample and long-term studies.

Of the 34 recurrent PTC patients in the group, postoperative recurrence intervals ranged from five months to 18 years, with a median time of 46 months. Recurrence was observed to occur within a short period of time. The majority of PTC patients, for example, showed recurrence within two to five years from surgery. Furthermore, recurrence may also occur more than once. Therefore, PTC patients must have regular reexamination with frequent follow-ups within five years after the first treatment. Ultrasound examination must be performed at least once a year within five years after the first treatment for timely discovery of tumor recurrence.

\section{Conclusions}

In conclusion, initial surgery approach, tumor size, extrathyroid invasion, lymph node metastases, and pathological type may be related to PTC recurrence. However, opinions on the resection scope of PTC differ. Surgical indications and methods vary on a case-to-case basis. According to Untch et al. [21], lobectomy for patients with low risk is suitable. Nixon et al. [22] demonstrated no difference in the 10-year survival rate of patients with low-risk highly differentiated thyroid carcinoma and those receiving lobectomy and total thyroidectomy. Doctors must consider patient compliance during follow-ups when evaluating whether or not the patient must receive thyroid lobectomy. For individuals with poor economic means, low education levels, poor knowledge about their condition, and inability to accomplish regular follow-up, complete thyroidectomy may be a suitable option. The most appropriate resection scope for PTC can be determined by considering the thoroughness of operation, the functions of the thyroid and parathyroid glands, and the relationships between important anatomical positions. Enhanced follow-up and postoperative reexamination must be carried out in patients with increased risk of recurrence. Effective and regular treatments must be administered once recurrence is discovered.

\section{Consent}

Written informed consent was obtained from the patient for the publication of this report and any accompanying images.

\section{Competing interests}

The authors declare that they have no competing interests.

\section{Authors' contributions}

$J Z$ and PFL conceived and designed the experiments. JZ, XLW and XXZ collected the data. PFL and HFH analyzed the data. PFL and XLW wrote the paper. All authors read and approved the final manuscript.

\section{Acknowledgments}

This study was supported by the President Fund of the General Hospital of Jinan Military Command (2013ZD01).

\section{Author details}

'Department of Thyroid and Breast Surgery, General Hospital of Jinan Military Command, Jinan 250031, China. ${ }^{2}$ Department of Pathology, Affiliated Hospital of Taishan Medical College, Taian 271000, China. ${ }^{3}$ Medical Administration Division, General Hospital of Jinan Military Command, Jinan 250031, China. ${ }^{4}$ Department of Pathology, General Hospital of Jinan Military Command, 25 Shifan Road, Tianqiao District, Jinan 250031, China.

${ }^{5}$ Department of Statistics, Taishan Medical College, Taian 271000, China.

Received: 20 January 2015 Accepted: 9 July 2015

Published online: 14 July 2015

\section{References}

1. Giordano D, Frasoldati A, Kasperbauer JL, Gabrielli E, Pernice C, Zini M, et al. Lateral neck recurrence from papillary thyroid carcinoma: predictive factors and prognostic significance. Laryngoscope. 2014. doi:10.1002/lary.25094.

2. Lee CW, Roh JL, Gong G, Cho KJ, Choi SH, Nam SY, et al. Risk factors for recurrence of papillary thyroid carcinoma with clinically node-positive lateral neck. Ann Surg Oncol. 2015;22(1):117-24.

3. Lang BH, Lee GC, Ng CP, Wong KP, Wan KY, Lo CY. Evaluating the morbidity and efficacy of reoperative surgery in the central compartment for persistent/recurrent papillary thyroid carcinoma. World J Surg. 2013;37(12):2853-9.

4. Londero SC, Krogdahl A, Bastholt L, Overgaard J, Trolle W, Pedersen HB, et al. Papillary thyroid microcarcinoma in Denmark 1996-2008: a national study of epidemiology and clinical significance. Thyroid. 2013;23(9):1159-64.

5. Ardito G, Revelli L, Giustozzi E, Salvatori M, Fadda G, Ardito F, et al. Aggressive papillary thyroid microcarcinoma: prognostic factors and therapeutic strategy. Clin Nucl Med. 2013;38(1):25-8.

6. Cooper DS, Doherty GM, Haugen BR, Kloos RT, Lee SL, Mandel SJ, et al. Revised American Thyroid Association management guidelines for patients with thyroid nodules and differentiated thyroid cancer. Thyroid. 2009;19(11):1167-214.

7. Mazzaferri EL, Jhiang SM. Long-term impact of initial surgical and medical therapy on papillary and follicular thyroid cancer. Am J Med. 1994;97(5):418-28.

8. Cunningham MP, Duda RB, Recant W, Chmiel JS, Sylvester JA, Fremgen A. Survival discriminants for differentiated thyroid cancer. Am J Surg. 1990;160(4):344-7.

9. Monacelli M, Lucchini R, Polistena A, Triola R, Conti C, Avenia S, et al. Total thyroidectomy and central lymph node dissection. Experience of a referral centre for endocrine surgery. G Chir. 2014;35(5-6):117-21.

10. Delogu D, Pisano IP, Pala C, Pulighe F, Denti S, Cossu A, et al. Prophylactic central neck lymphadenectomy in high risk patients with $\mathrm{T} 1$ or $\mathrm{T} 2$ papillary thyroid carcinoma: is it useful? Ann Ital Chir. 2014;85(3):225-9.

11. Delellis RA, Lloyd RV, Heitz PU, Eng C. Pathology and Genetics Tumours of Endocrine Organs. Lyon: IARC press; 2003.

12. Roman S, Sosa JA. Aggressive variants of papillary thyroid cancer. Curr Opin Oncol. 2013;25(1):33-8.

13. Kuo CS, Tang KT, Lin JD, Yang AH, Lee CH, Lin HD. Diffuse sclerosing variant of papillary thyroid carcinoma with multiple metastases and elevated serum carcinoembryonic antigen level. Thyroid. 2012;22(11):1187-90.

14. Evans WD. De-differentiation of papillary thyroid carcinoma into squamous cell carcinoma. A case of coexistence within an excised neck lesion. BMJ Case Rep 2012, 2012. doi: 10.1136/bcr-2012-007707.

15. Zidan J, Karen D, Stein M, Rosenblatt E, Basher W, Kuten A. Pure versus follicular variant of papillary thyroid carcinoma: clinical features, prognostic factors, treatment, and survival. Cancer. 2003;97(5):1181-5.

16. Melcescu E, Horton WB, Pitman KT, Vijayakumar V, Koch CA. Euthyroid Graves' orbitopathy and incidental papillary thyroid microcarcinoma. Hormones (Athens). 2013;12(2):298-304. 
17. Boone RT, Fan CY, Hanna EY. Well-differentiated carcinoma of the thyroid. Otolaryngol Clin North Am. 2003;36(1):73-90. viii.

18. Shoup M, Stojadinovic A, Nissan A, Ghossein RA, Freedman S, Brennan MF, et al. Prognostic indicators of outcomes in patients with distant metastases from differentiated thyroid carcinoma. J Am Coll Surg. 2003;197(2):191-7.

19. Ito Y, Tomoda C, Uruno T, Takamura Y, Miya A, Kobayashi K, et al. Clinical significance of metastasis to the central compartment from papillary microcarcinoma of the thyroid. World J Surg. 2006;30(1):91-9.

20. Watkinson JC, Franklyn JA, Olliff JF. Detection and surgical treatment of cervical lymph nodes in differentiated thyroid cancer. Thyroid. 2006;16(2):187-94.

21. Untch BR, Palmer FL, Ganly I, Patel SG, Michael Tuttle R, Shah JP, et al. Oncologic outcomes after completion thyroidectomy for patients with well-differentiated thyroid carcinoma. Ann Surg Oncol. 2014;21(4):1374-8.

22. Nixon IJ, Ganly I, Patel SG, Palmer FL, Whitcher MM, Tuttle RM, et al. Thyroid lobectomy for treatment of well differentiated intrathyroid malignancy. Surgery. 2012;151(4):571-9.

\section{Submit your next manuscript to BioMed Central and take full advantage of:}

- Convenient online submission

- Thorough peer review

- No space constraints or color figure charges

- Immediate publication on acceptance

- Inclusion in PubMed, CAS, Scopus and Google Scholar

- Research which is freely available for redistribution 